\title{
A NOTE ON MOUFANG VEBLEN-WEDDERBURN SYSTEMS
}

\author{
M. L. NARAYANA RAO
}

\begin{abstract}
The purpose of this note is to show that a VeblenWedderburn system with multiplicative Moufang identity is a near field if its dimension $d$ over its kern does not exceed 7 .
\end{abstract}

1. Introduction. In a recent paper [2] Kallaher investigates (left) Veblen-Wedderburn systems (and the corresponding projective planes) in which the Moufang identity

$$
(x \cdot y) \cdot(z \cdot x)=(x \cdot(y \cdot z)) \cdot x
$$

holds. Such a system is called a Moufang (left) Veblen-Wedderburn system (MVW system). Fields, near fields and Cayley-Dickson algebras are examples of MVW systems. A proper MVW system is one in which the other distributive law does not hold. The only proper MVW systems known are the near fields. Kallaher [2] obtains two sets of conditions under which an MVW system is a near field. Recently the author has been able to show [3] that there are no proper finite MVW systems other than the near fields. The object of this note is to extend this result to the infinite systems of dimension $d \leqq 7$ over their kerns.

2. During the course of the proof of Theorem 1 the following results are needed. Proofs of these results may be found in the references indicated.

Result 1. Every two elements of a Moufang loop generates a subgroup (di-associativity) (Bruck [1]).

Result 2. Let $F(+, \cdot)$ be an MVW system with kern $K$. If $A(\cdot)$ is a maximal associative subloop contained in the loop $F^{\prime}(\cdot)$, then $B(+, \cdot)$ is a maximal near field contained in $F(+, \cdot)$ where $B$ $=A \cup\{0\}$ and $F^{\prime}$ consists of all nonzero elements from $F$. Further $B$ contains $K$ [3, Lemmas 3.1 and 3.2].

THEOREM 1. Let $F(+, \cdot)$ be a left MVW system of dimension $d$ over its kern $K$ (as a right vector space). If $d \leqq 7$, then $F(+, \cdot)$ is a near field.

Received by the editors July 10, 1969.

AMS Subject Classifications. Primary 2098, 1700; Secondary 5070.

Key Words and Phrases. Veblen-Wedderburn systems, Moufang identity, projective planes, near fields, power associativity, di-associativity, associativity. 
ProOF. In the course of this proof we use freely the right inverse property, left distributive law and some properties of the kern $K$. Also we write $a b$ in place of $a \cdot b$. If $d=1$, then $F=K$ and the theorem is obvious. Suppose $1<d \leqq 7$. Let $x$ be an element from $F$ which does not belong to $K$ and $G=\langle x\rangle$ be the subloop of $F^{\prime}(\cdot)$ generated by $x$. $G$ is obviously an associative subloop of $F^{\prime}(\cdot)$ and consequently there exists a maximal associative subloop $A$ in $F^{\prime}(\cdot)$ which contains $G$. From Result 2 it follows that $B(+, \cdot)$ is a maximal near field where $B=A \cup\{0\}$. If $B=F$, the theorem is proved. Suppose $B<F$. Then there is an element $y$ in $F$ such that $y \notin B$. Let $H=\langle x, y\rangle$, the subloop of $F^{\prime}(\cdot)$ generated by $x$ and $y$ ( $H$ exists since $F^{\prime}(\cdot)$ is di-associative). Let $M$ be a maximal associative subloop of $F^{\prime}(\cdot)$ containing $H$. Using Result 2 again we obtain that $N(+, \cdot)$ is a near field where $N$ $=M \cup\{0\}$. We claim that $N=F$ and consequently $F(+, \cdot)$ is a near field. Suppose $N<F$. Then there is an element $z$ in $F$ which does not belong to $N$. We now show that the existence of $z$ leads to the conclusion that the set $T=\{1, x, y, y x, z, z x, z y, z(y x)\}$ is independent over $K$ implying a contradiction that $F(+, \cdot)$ is of dimension $d \geqq 8$ over its kern. Firstly we show that the set $\{1, x, y, y x\}$ is independent over $K$. Suppose there are elements $a, b, c$ and $k$ in $K$ such that $a+x b$ $+y c+y x k=0$. Then it follows that $y(c+x k)=-(a+x b)$. Suppose $c+x k \neq 0$. Then $y=(-(a+x b))(c+x k)^{-1} \in B$ a contradiction to the choice of $y$. Thus $a+x b=0$ and consequently $c+x k=0$ which imply that $a=b=c=d=0$. Hence the set $\{1, x, y, y x\}$ is independent over $K$.

Suppose there are elements $a, b, c, k, e, f, g$ and $h$ in $K$ such that $a+x b+y c+y x k+z e+z x f+z y g+z(y x) h=0$. This relation may be rewritten as $z X=Y$ where $X=(e+x f+y g+y x h)$ and $Y$ $=-(a+x b+y c+y x k)$. Suppose $X \neq 0$. Then $z=Y X^{-1} \in N$ a contradiction to the choice of $z$. Hence $X=0$ and consequently $Y=0$. Since the set $\{1, x, y, y x\}$ is independent over $K$, we obtain that $a=b=c$ $=k=e=f=g=h=0$. Thus $T$ is an independent set over $K$. This completes the proof of the theorem.

The question of existence of infinite proper MVW systems of dimension $d$ over their kerns for $d \geqq 8$ still remains unresolved.

\section{REFERENCES}

1. R. H. Bruck, A survey of binary systems, Ergebnisse der Mathematik und ikrer Grenzgebiete, Heft 20, Springer-Verlag, Berlin, 1958. MR 20 \#76.

2. M. J. Kalaher, Moufang Veblen-Wedderburn systems, Math. Z. 105 (1968), 114127. MR 37 \#3438.

3. M. L. Narayana Rao, A question on finite Moufang Veblen-Wedderburn systems, J. Algebra (to appear).

UNIVERSITY OF MISSOURI 